\title{
pneumonia
}

Case Report

\section{Acute fibrinous and organising pneumonia following lung transplantation is associated with severe allograft dysfunction and poor outcome: a case series}

\author{
Keith C Meyera, Jennifer Bierach ${ }^{b}$, Jeffrey Kanne', Jose R Torrealba ${ }^{d}$, Nilto C De Oliveirae \\ aSection of Allergy, Pulmonary, and Critical Care Medicine, Department of Medicine. University of Wisconsin School of Medicine and Public Health, \\ Madison, Wisconsin, USA; bUtica Park Clinic, Tulsa, Oklahoma, USA; 'Department of Radiology, University of Wisconsin School of Medicine and \\ Public Health, Madison, Wisconsin, USA; 'Department of Pathology, University of Texas Southwestern Medical Center, Dallas, Texas, USA; ${ }^{\text {SSection of }}$ \\ Cardiothoracic Surgery, Department of Surgery, University of Wisconsin School of Medicine and Public Health, Madison, Wisconsin, USA
}

Corresponding author: Keith C Meyer, Professor of Medicine, University of Wisconsin Lung Transplant and Advanced Lung Disease Program, Section of Allergy, Pulmonary and Critical Care Medicine, Department of Medicine, University of Wisconsin School of Medicine and Public Health, K4/910 Clinical Science Center, 600 Highland Avenue, Madison, Wisconsin, 53792-9988, USA. Phone: 608 263-6363 (office); 608 263-3035 (secretary) Email: kcm@medicine.wisc.edu

\begin{abstract}
Author contributions: All the authors met ICMJE authorship criteria. KM conceived of and contributed to the study design. KM, JB and NDO contributed to data collection, data interpretation, and manuscript composition. JK and JT contributed to image selection and production, data interpretation, and manuscript composition. KM wrote the first draft of the manuscript. All authors critically reviewed the manuscript for important intellectual content. All authors agree with the manuscript results and conclusions.
\end{abstract}

Received 4 April 2015; Accepted 23 June 2015; Published 1 September 2015

Citation: Meyer KC, Bierach J, Kanne J, Torrealba JR, De Oliveira NC. Acute fibrinous and organising pneumonia following lung transplantation is associated with severe allograft dysfunction and poor outcome: a case series. pneumonia 2015;6:67-76

\begin{abstract}
Acute fibrinous and organising pneumonia (AFOP) is a histopathologic variant of acute lung injury that has been associated with infection and inflammatory disorders and has been reported as a complication of lung transplantation. A retrospective chart review was performed for all patients transplanted at the University of Wisconsin Hospital and Clinics from January 1995 to December $2013(n=561)$. We identified 6 recipients whose clinical course was complicated by AFOP. All recipients were found to have AFOP on lung biopsy or at post-mortem examination, and 5 of the 6 patients suffered progressive allograft dysfunction that led to fatal outcome. Only 1 of the 6 patients stabilised with augmented immunosuppression and had subsequent improvement and stabilisation of allograft function. We could not clearly identify any specific cause of AFOP, such as drug toxicity or infection. Lung transplantation can be complicated by lung injury with an AFOP pattern on histopathologic examination of lung biopsy specimens. The presence of an AFOP pattern was associated with irreversible decline in lung function that was refractory to therapeutic interventions in 5 of our 6 cases and was associated with severe allograft dysfunction and death in these 5 individuals. AFOP should be considered as a potential diagnosis when lung transplant recipients develop progressive decline in lung function that is consistent with a clinical diagnosis of chronic lung allograft dysfunction.
\end{abstract}

Keywords: pneumonia, interstitial, acute fibrinous and organising pneumonia, lung transplantation, thoracic 


\section{Introduction}

Acute fibrinous and organising pneumonia (AFOP) appears to be a variant of acute lung injury ( $A L I$ ) that has characteristics of diffuse alveolar damage (DAD) as well as features of organising pneumonia (OP) [1]. It has a histopathological pattern that is characterised by intra-alveolar fibrin associated with mononuclear cell infiltrates and loose intraluminal connective tissue with a patchy distribution. AFOP lacks the hyaline membranes that are classically seen in the subacute phase of DAD; it does not have intra-bronchiolar fibrosis (as seen in $\mathrm{OP}$ ), or prominent infiltration with eosinophils (as seen in eosinophilic pneumonia). AFOP has been linked to various conditions including infections, drug toxicity, and occupational exposures.

Lung transplant recipients are at high risk for both infection and lung allograft rejection. The most significant barrier to long-term survival following lung transplantation is susceptibility to chronic lung allograft dysfunction (CLAD), the predominant cause of which is obliterative bronchiolitis (OB) that leads to bronchiolitis obliterans syndrome (BOS). BOS is characterised by airflow obstruction that usually progresses over time, and is the leading cause of allograft loss and recipient death $[2,3]$. However, allograft pathologies other than BOS/OB have also been reported as a cause of CLAD. These include restrictive allograft syndrome (RAS), which has been associated with DAD as a precursor lesion and leads to a restrictive pattern of allograft dysfunction with a prognosis that is worse than that for CLAD caused by BOS $[4,5]$. More recently, Paraskeva et al. [6] identified 87 recipients in a cohort of 194 bilateral lung transplant recipients who developed CLAD; 22 of these patients had a restrictive pattern of allograft dysfunction and were found to have AFOP by histopathologic and radiographic criteria. The recipients who developed AFOP had significantly worse post-transplant survival than a cohort of 39 recipients who developed BOS/OB.

We report on 6 lung transplant recipients who developed severe CLAD that was associated with an AFOP histopathologic pattern on lung biopsy and/or autopsy specimens.

\section{Methods}

We retrospectively reviewed the records (prospectively collected and recorded data in the University of Wisconsin Hospital and Clinics lung transplant database) of all lung and heart-lung recipients who were transplanted at our centre from January 1995 to December 2013. None of the recipients had pre-transplant evidence of sensitisation. All recipients had routine clinical evaluations that included chest imaging (posterior-anterior and lateral view chest radiograph $[C X R]$ ) and spirometry performed at least every 6 months following lung transplant. Transbronchial and, on occasion (if needed), surgical lung biopsies were performed when suspected allograft dysfunction was detected, and the histopathologic findings from all biopsy specimens were also entered into the database.

All recipients were placed on maintenance immune suppression using tacrolimus, mycophenolate, and corticosteroids, and all received prophylactic antiviral therapy for cytomegalovirus and imidazole antifungal prophylaxis.

All lung transplant recipients received surveillance bronchoscopies with bronchoalveolar lavage (BAL) and transbronchial biopsies (TBBs) to detect acute cellular rejection and/or lymphocytic bronchiolitis with grading according to criteria set by The International Society for Heart \& Lung Transplantation (ISHLT) Lung Rejection Study Group [7] at post-transplant weeks 2, 6, 12, 18, 26, 40, and 52. Outpatient clinic evaluations, routine chest imaging, and pulmonary function testing were also performed at these time intervals during the first year.

Clinical evaluations, radiographic imaging, pulmonary function testing, and bronchoscopies were also performed whenever necessary for clinical indications, with follow-up bronchoscopies performed 4 weeks after a previous bronchoscopy where acute rejection of any grade or other significant allograft problem was detected. Thoracic high-resolution computed tomography (HRCT) was obtained if clinically indicated. All biopsy results, thoracic imaging, BAL culture data, and pulmonary function test results were retrospectively reviewed for this study. Screening for donor specific antibodies was not conducted at the time when these recipients were diagnosed with AFOP.

\subsection{Ethics statement}

This investigation was approved by the University of Wisconsin Human Subjects Committee (Approval number: M-2009-1308).

\section{Results}

The cohort consisted of a total of 561 lung transplant recipients. Of these, 6 recipients were diagnosed as having CLAD due to AFOP. Clinical and demographic characteristics of these 6 patients are given in Table 1, and pertinent data from diagnostic bronchoscopy with BAL and histopathologic findings are given in Table 2. One patient (Case 2) had the diagnosis made via surgical lung biopsy (Figure 1C), and a repeat wedge biopsy from this patient 1 month later showed evidence of persistent AFOP, but prominent polymorphonuclear leukocyte infiltration with fibrin deposition and organising pneumonia (likely due to bacterial infection) was also seen on the second biopsy. The other 5 patients had adequate tissue sampling with TBB that allowed the diagnosis to be made pre-mortem. The diagnosis of AFOP was made at autopsy for Case 3. 


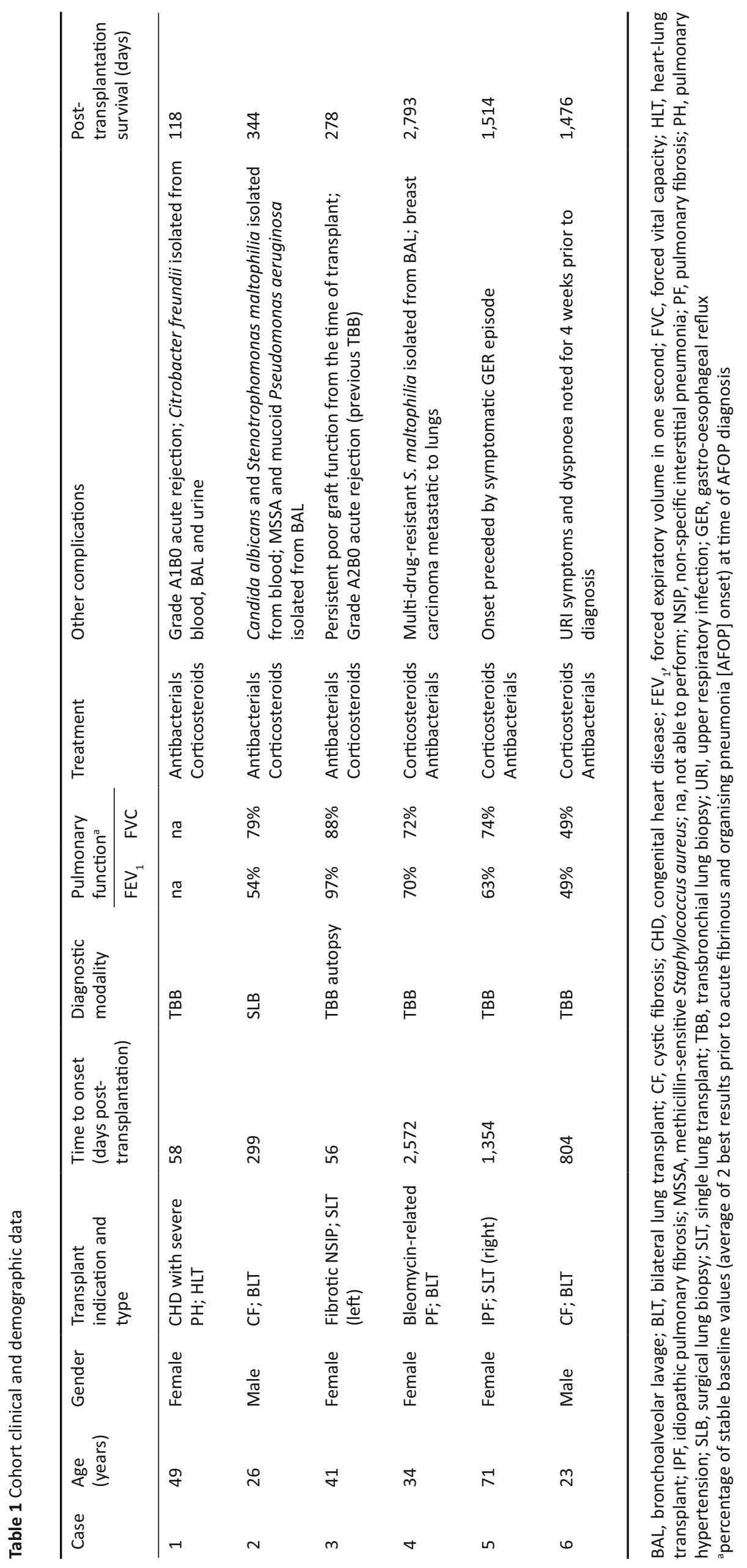




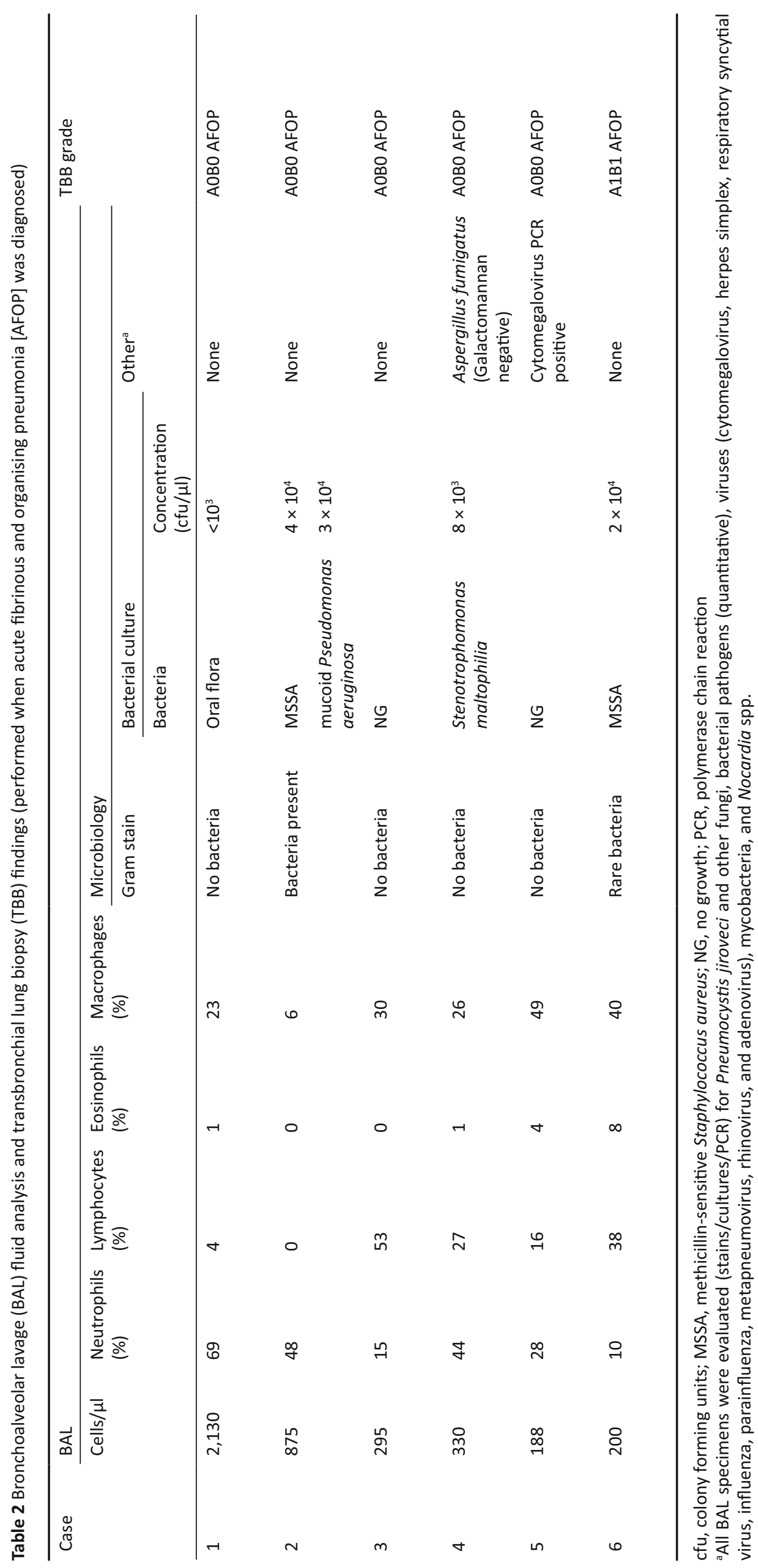




\section{Case series}

\subsection{Case 1}

A 48-year-old female received a heart-lung transplant for congenital heart disease. Early sputum cultures demonstrated Gram-negative rods for which piperacillin-tazobactam was given, and she was extubated on the second day, post-transplant, and commenced ambulation. However, small bowel complications developed and required surgical resection, and she developed dialysis-dependent acute renal insufficiency. Intubation and mechanical ventilation were required on day 30, and chest imaging showed persistent diffuse infiltrates (Figure 1, A and B). TBB showed minimal acute rejection (Grade $A 1 B 0)$. She was extubated 9 days later but required reintubation (post-transplant day 58) for progressive respiratory failure. TBB biopsy showed AFOP but did not show acute rejection (Figure $2, A$ and $B$ ), and an endomyocardial biopsy was negative for cardiac rejection. A laparotomy and right hemicolectomy were performed for persistent volvulus (post-transplant day 100). Lung function worsened, and Citrobacter freundii was isolated from blood cultures, BAL, and urine cultures. Respiratory status progressively worsened, and life support was withdrawn.

\subsection{Case 2}

A 26-year-old man received a successful bilateral lung transplant for end-stage cystic fibrosis but required stent placement for a right main bronchial stenosis at the anastomosis site. He developed cough, dyspnoea, and fever 5 months post-transplantation; sputum cultures demonstrated mucoid Pseudomonas aeruginosa for which piperacillin-tazobactam was administered. TBB showed minimal acute rejection (Grade A1B0). Cough and dyspnoea persisted and the patient resumed use of supplemental home oxygen. He acutely decompensated 2 weeks later with diffuse infiltrates on thoracic imaging (Figure 1, C) and developed shock and respiratory insufficiency, requiring intensive care unit admission and administration of pressors, tracheal intubation, and mechanical ventilation. Group A Streptococcus pyogenes was present in multiple blood cultures, and diabetic ketoacidosis was also present. He was successfully treated, extubated, and discharged to home 12 days after admission. After a 3-month period of clinical stability, he developed sustained decline in forced expiratory volume in 1 second $\left(F_{1} V_{1}\right)$. BAL showed methicillin-sensitive Staphylococcus aureus (MSSA) and mucoid $P$. aeruginosa. TBB did not show acute rejection or other specific parenchymal abnormality. Antibiotics were administered, and blood cultures grew Candida albicans and Stenotrophomonas maltophilia. Intubation and mechanical ventilatory support were required. Due to lack of improvement with appropriate antibacterial and anti-fungal therapies, a surgical lung biopsy was performed that showed AFOP (Figure 2, C and D). Chest imaging showed persistent infiltrates, and care was withdrawn after 1 month of mechanical ventilation.

\subsection{Case 3}

A 41-year-old female received a left single lung transplant for fibrotic non-specific interstitial pneumonia (NSIP). At 2 weeks post-transplant surveillance, TBB specimens showed Grade A1B1 acute rejection, and TBB performed 2 weeks later showed Grade $\mathrm{A} 2 \mathrm{~B} 0$ acute rejection. Intravenous methylprednisolone burst and taper was given, and she remained asymptomatic and continued to improve clinically until 6 weeks post-transplant when she presented with increasing dyspnoea on exertion, cough, and low-grade fever that progressed to intubation and mechanical ventilation. She was treated with broadspectrum antibacterial agents and pulse corticosteroids. TBB demonstrated organising pneumonia (OP) but no acute rejection, and $\mathrm{BAL}$ did not reveal any pathogens. She was successfully extubated after 4 days, weaned to room air, and discharged to home. However, subsequent surveillance TBBs showed persistent OP as well as acute rejection (Grade $\mathrm{A} 2-\mathrm{A} 3$ ), and total lymphoid irradiation was initiated. The patient was readmitted 6 weeks later for shortness of breath and hypoxaemia that progressed to respiratory failure, requiring mechanical ventilation. Despite broad-spectrum antibiotics and pulse corticosteroids, diffuse parenchymal opacities (Figure 1, D) progressively worsened, and supportive care was eventually withdrawn. AFOP was observed in lung specimens examined at autopsy (Figure 2, E and F).

\subsection{Case 4}

A 34-year-old woman who had been considered cured of Hodgkin's lymphoma underwent bilateral lung transplantation for bleomycin-associated pulmonary fibrosis. She had a benign clinical course for the next 7 years despite persistent poor graft function, but was then diagnosed with a left breast ductal carcinoma and underwent mastectomy and was started on tamoxifen. Three months later she noted persistent mild symptoms of cough and dyspnoea on exertion, associated with new persistent infiltrates on imaging (Figure 1, E and $\mathrm{F})$, accompanied by a significant decline in $\mathrm{FEV}_{1}$ and oxyhaemoglobin desaturation with activity. TBB revealed AFOP, and high-dose corticosteroids and broad-spectrum antibiotics were given. BAL detected multi-drug resistant S. maltophilia, for which antibacterial coverage was adjusted. She gradually improved and was discharged to home, but after a stable period of 6 months, she developed increased shortness of breath, and a thoracic HRCT showed multiple new lung nodules. Lung biopsy revealed metastatic breast cancer, to which she succumbed 3 months later.

\subsection{Case 5}

A 67-year-old female received a right single lung transplant for idiopathic pulmonary fibrosis and had an uncomplicated post-transplant course; surveillance bronchoscopies did not show acute rejection or infection, and 4 months post-transplantation her $\mathrm{FEV}_{1}$ reached a zenith of $129 \%$ 


\section{Figure 1A}

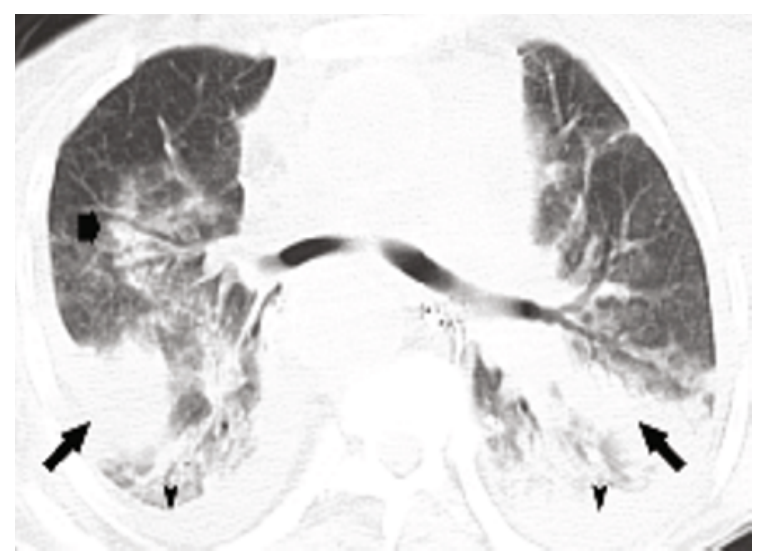

Figure 1C

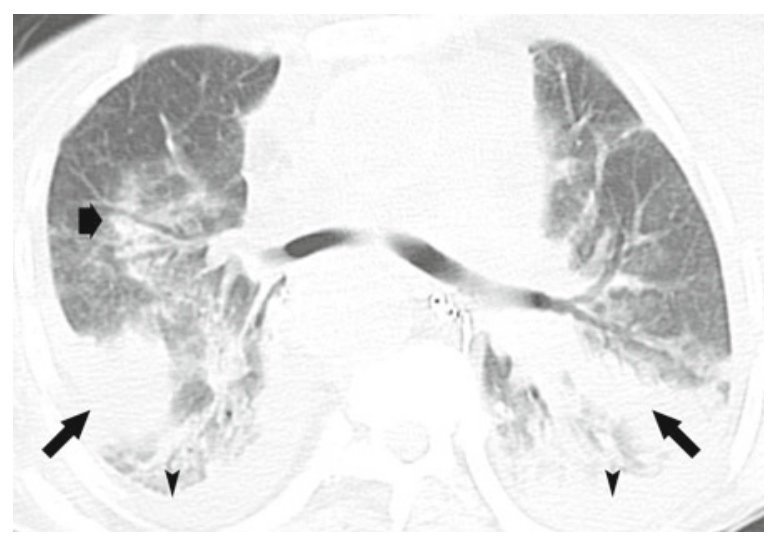

\section{Figure 1E}

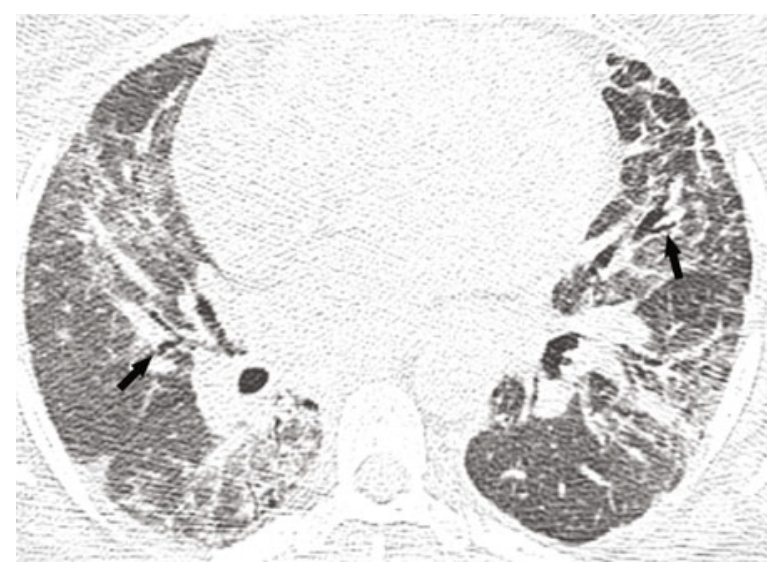

Figure 1B

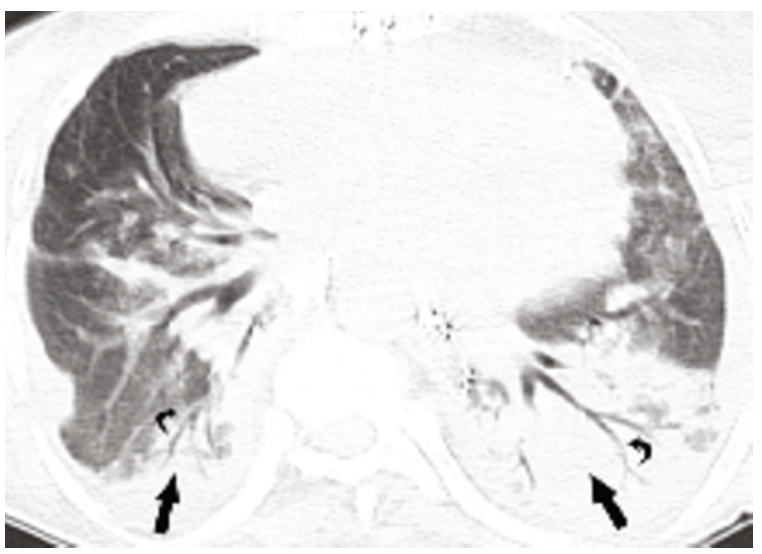

Figure 1D

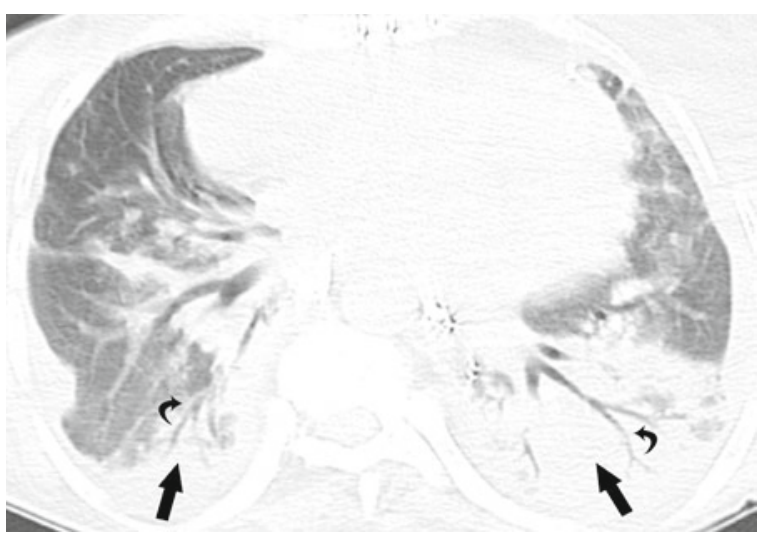

Figure 1F

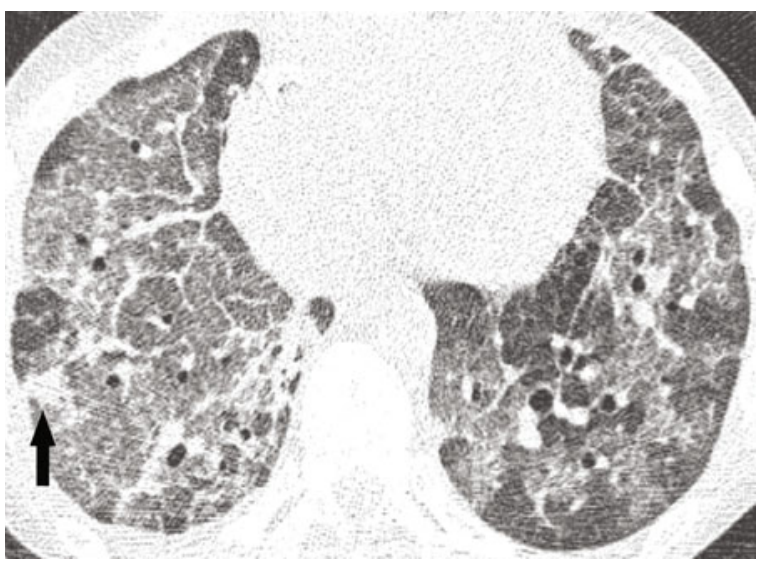

Figure 1 Case 1: Transverse unenhanced computed tomography (CT) images ( $A$ and B) demonstrate dense peribronchial consolidation (thin arrows) with air bronchograms (curved arrows); note the rim of consolidation (wide arrow) peripheral to a focus of ground-glass opacity, reminiscent of the "reverse halo" sign seen in organising pneumonia, and small bilateral effusions are present (arrowheads). Case 2: Transverse section (C) shows several foci of peribronchial and perilobular consolidations (arrows) and ground-glass opacity (arrowheads), and small pleural effusions are present. Case 3: Transverse high-resolution computed tomography (HRCT) image (D) demonstrates patchy peribronchial ground-glass opacity with superimposed reticulation and mild traction bronchiectasis (arrows). Case 4: Transverse HRCT images (E and F) show extensive, patchy ground-glass opacity, traction bronchiectasis (arrows in E), superimposed reticulation with peripheral and peribronchial consolation $(F)$, and interlobular septal thickening (F). 


\section{Figure 2A}

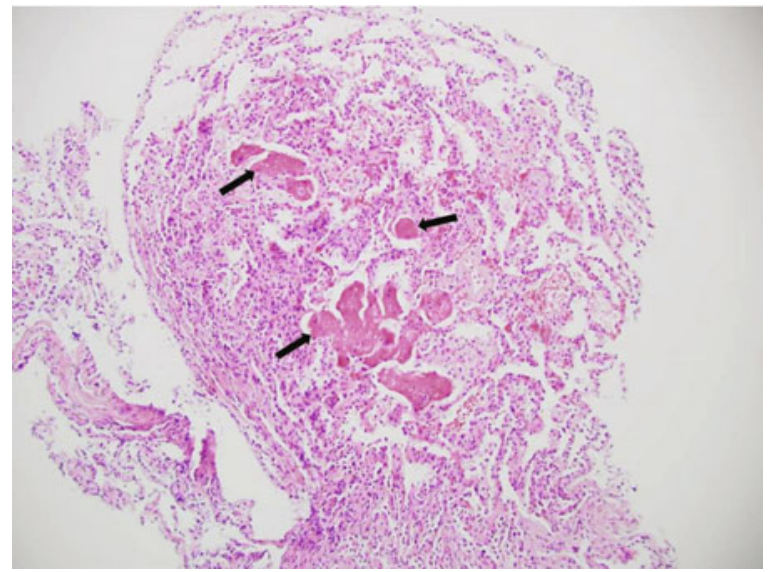

\section{Figure 2C}

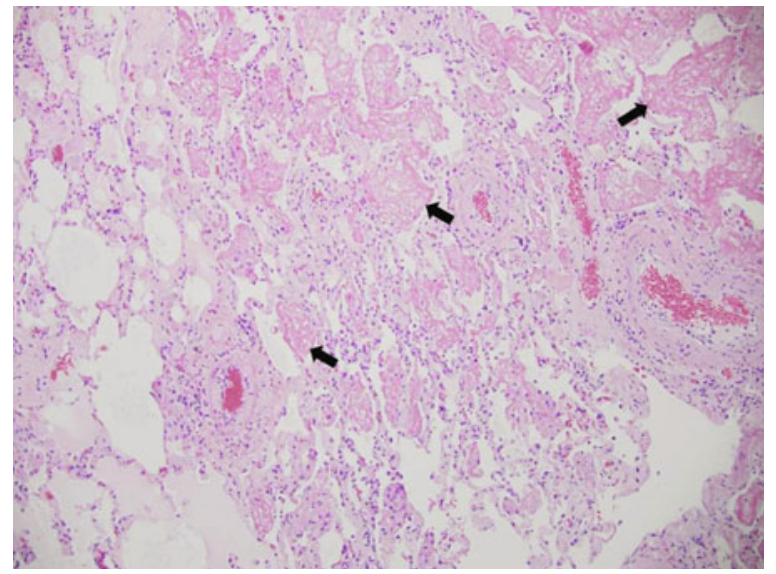

\section{Figure 2E}

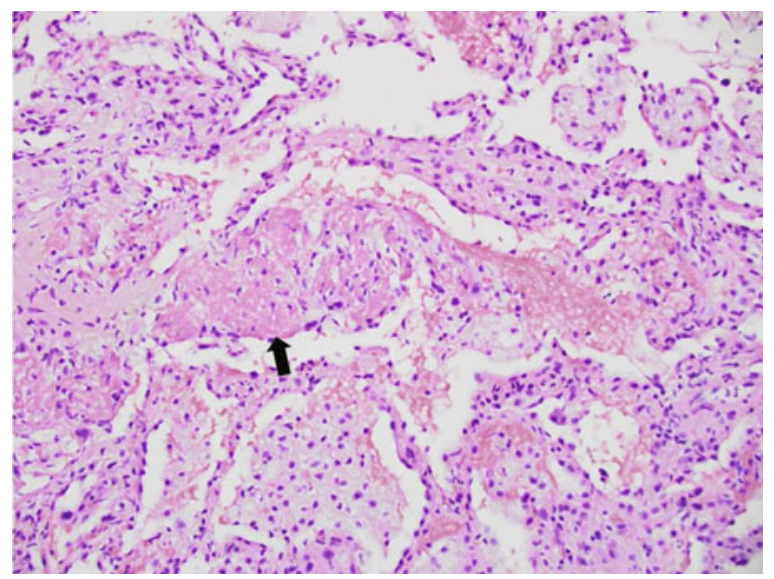

\section{Figure 2B}

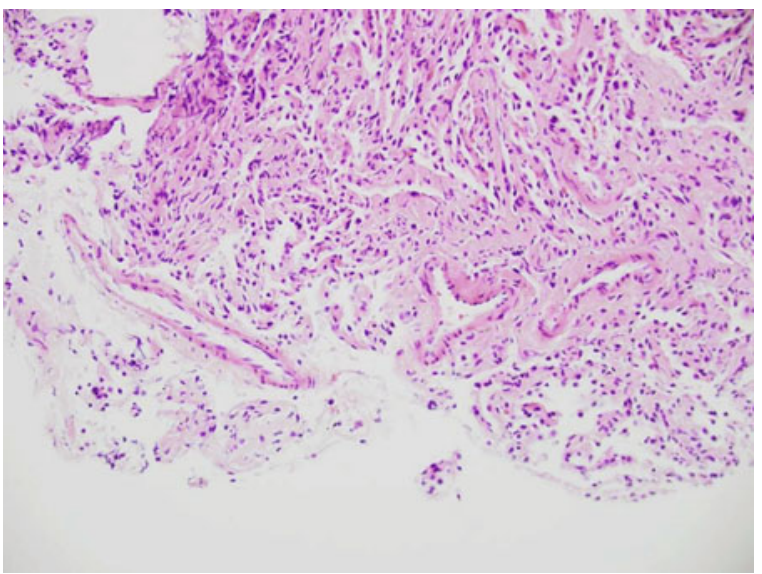

\section{Figure 2D}

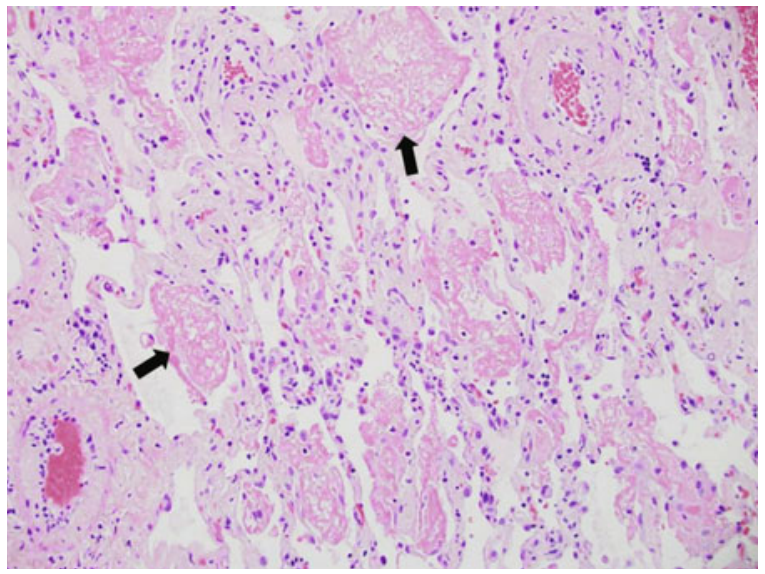

\section{Figure 2F}

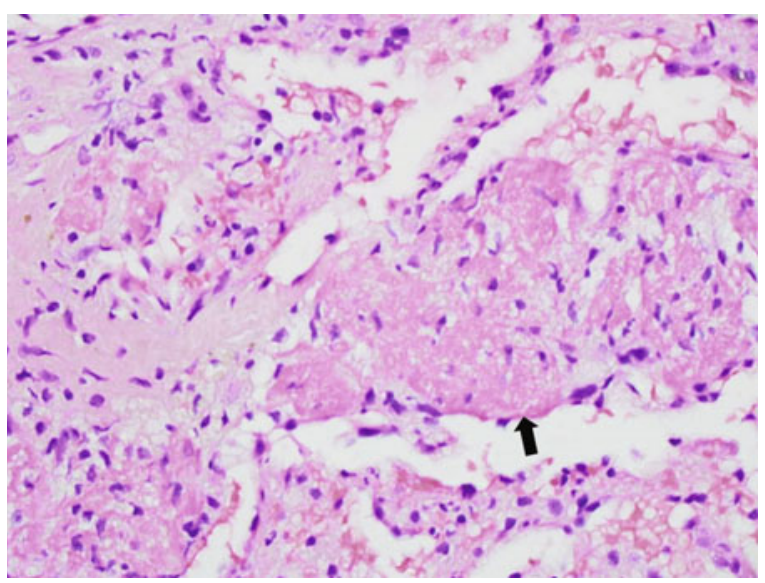

Figure 2 Case 1: Transbronchial biopsy shows lung parenchyma with intra-alveolar fibrin aggregates or "balls" (arrows) with mild mononuclear interstitial infiltrate (A: Haematoxylin-eosin stain $[\mathrm{H} / \mathrm{E}], 100 \times$ magnification), without evidence of cellular rejection given the lack of perivascular or bronchial mucosal infiltrates (B: H/E, 200× magnification). Case 2: Surgical lung biopsy shows intraalveolar fibrin aggregates (arrows) and mild fibroblastic proliferation with minimal mononuclear inflammatory cell infiltrate (C: $\mathrm{H} / \mathrm{E}$ 100x magnification; D: H/E 200x magnification). Case 3: Autopsy specimen shows intra-alveolar fibrin aggregates (arrows) (E: H/E 100x magnification; F: H/E 200x magnification). 
of predicted. She remained stable for the next 44 months until diagnosed with BOS (Stage 1). Chest imaging revealed new patchy consolidation in the allograft. Bronchoscopy revealed BAL neutrophilia (28\%), and cytomegalovirus was detected in BAL (but not peripheral blood) via polymerase chain reaction. TBB was negative for acute rejection or cytomegalovirus pneumonitis, but showed changes of AFOP. Intravenous pulse methylprednisolone and antibiotics were administered with gradual clearing of the allograft infiltrates on CXR. However, FEV continued to decline, and follow-up bronchoscopy 2 months later revealed persistent AFOP and BAL neutrophilia (56\%), despite clearing of the allograft infiltrates on CXR. BAL was

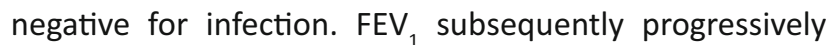
declined and the patient eventually succumbed to respiratory failure.

\subsection{Case 6}

A 22-year-old male received a bilateral lung transplant for cystic fibrosis and had an essentially uncomplicated post-transplantation course for 26 months (with the exception of a Grade A2 rejection episode at 12.5 months post-transplant). He developed worsening cough, increasing fatigue, and dyspnoea following exposure to his spouse who had developed upper respiratory tract infection symptoms. Spirometry showed a significant decline in $\mathrm{FEV}_{1}$, and oxyhaemoglobin desaturation was noted. TBB revealed changes of AFOP and was also graded as showing a Grade A1B1 acute rejection, and BAL cultured MSSA at $2 \times 10^{4}$ colony forming units $(\mathrm{cfu}) / \mu$ l but was negative for respiratory viruses. The patient's symptoms initially improved with antibiotics and pulse methylprednisolone, but $\mathrm{FEV}_{1}$ subsequently progressively declined and the patient eventually succumbed to respiratory insufficiency.

\section{Discussion}

Beasley and colleagues [1] described AFOP as a new histopathologic entity in 2002 and concluded that it represented an unusual variant of ALI. OP is characterised by buds of granulation tissue (comprised of fibroblasts and myofibroblasts in a matrix of loose connective tissue) in distal airspaces (alveolar ducts, alveoli, and bronchioles) that may be idiopathic (cryptogenic organising pneumonia [COP]) [8,9]. OP has been associated with a variety of clinical entities such as infection, connective tissue disorders, or pneumotoxic drug reactions [9]. DAD has also been associated with a number of clinical disorders such as infection or drug toxicity; it is characterised by alveolar epithelial cell and endothelial injury with fluid and cellular exudation followed by reparative fibroblast proliferation and alveolar type II pneumocyte hyperplasia [10]. Hyaline membrane formation marks the exudative phase of DAD. AFOP is characterised histopathologically by intra-alveolar fibrin (in the form of fibrin "balls") within the alveolar spaces [11]. The presence of the fibrin balls differentiates AFOP from classical OP (with its granulation tissue in the bronchioles, alveolar ducts, and alveoli) as well as from DAD (with its classic hyaline membranes). Patchy distribution of fibrin deposition is typical and involves $25-90 \%$ of alveolar spaces, averaging $50 \%$ of airspace involvement overall. In contrast to AFOP, DAD is typically diffuse. Although AFOP lacks the classic hyaline membranes that characterise DAD, myxoid fibroblastic tissue has been identified in the alveolar walls in both AFOP and DAD. Eosinophils are typically present, but not predominant as with eosinophilic pneumonitis.

Routine chest radiographs of patients with AFOP typically show interstitial changes and may show areas that appear consolidated $[1,12]$. All of our patients had diffuse, persistent infiltrates on thoracic imaging. HRCT imaging (Figure 2) showed peribronchial and perilobular consolidation with areas of ground-glass opacity. Bronchoalveolar lavage performed at the time of diagnosis of AFOP (Table 2) showed considerable BAL neutrophilia for all patients, but only 2 patients had bacteria visualised on Gram stain at $\geq 1 \times 10^{4} \mathrm{cfu} / \mu$ l of BAL fluid.

Many clinical conditions have been associated with AFOP, and AFOP has been linked to drug reactions (e.g. decitabine, amiodarone, abacavir), infections (e.g. severe acute respiratory syndrome, respiratory syncytial virus, Haemophilus influenzae, Acinetobacter), and various forms of connective tissue disorders [1,13,14]. Other associations include stem cell transplantation, haematologic malignancy, occupational exposures, and cigarette smoking $[13,14]$. Although AFOP often runs a rapid and fatal course, responses to pharmacologic interventions have been reported. Treatments associated with significant clinical responses include corticosteroids, cyclophosphamide, and mycophenolate [15]. Acute onset accompanied by the need for mechanical ventilation appears to correlate with a poor prognosis [1].

Infection and/or drug toxicity may have played a key role in AFOP pathogenesis in our patients. Another possibility is that AFOP represented a form of allograft rejection, although these patients had ongoing intense immunosuppression and various complications associated with their lung transplant status, and 3 of the 6 patients did not have improvement with intensified immune suppression. None of our subjects received drugs that have been linked to AFOP in published literature. Additionally, none of the surgical or TBB specimens revealed a consistent association with acute rejection or lymphocytic bronchiolitis. Because of the intense immunosuppression and multiple drugs that our lung transplant recipients were receiving, plus the numerous drugs (many associated with potential pulmonary toxicity) they were taking for management of their post-transplant issues as well as ongoing colonisation and/or infection with potential pathogens, it is difficult to associate AFOP with any specific cause. Only 1 of our 6 patients stabilised and appeared to recover. Her dyspnoea, infiltration on chest radiographs, and gas exchange were rapidly worsening, but she stabilised and gradually improved when intensified immunosuppression (intravenous methylprednisolone and subsequent gradual prednisone taper) was given. A 
point of interest is the infrequent occurrence of AFOP in our recipient population (6 of 561 [1\%]) as compared to the report by Paraskeva et al. (22 of 194 [11\%]) [6].

Sato et al. [4] used a $\geq 10 \%$ decline in baseline total lung capacity (TLC) to indicate the presence of restrictive physiology in addition to the presence of radiographic infiltrates on chest imaging and DAD on histopathology as diagnostic of the RAS form of CLAD. Although we did not measure lung volumes in our cohort of recipients with AFOP, the clear decline of both $\mathrm{FEV}_{1}$ along with forced vital capacity (FVC) in 4 of the 6 subjects is consistent with restrictive physiology. Because most centres do not measure lung volumes and many factors can confound measurements of TLC, we suggest that the combination of spirometry, imaging findings, and histopathology can be used to identify AFOP and differentiate it from the RAS or the classical obstructive BOS/OB phenotypes of CLAD [3]. Our experience plus that reported by Paraskeva et al. [6] indicates that AFOP should be considered as a diagnosis in transplant recipients who develop allograft dysfunction when clinical evaluation suggests that CLAD associated with restrictive physiology and radiographic infiltrates has developed. Although upper lobe infiltrates in this setting are highly suggestive of a diagnosis of RAS [4,6], lung biopsy is required to differentiate AFOP from RAS.

In conclusion, we identified 6 lung transplant recipients who developed diffuse infiltrates and had histopathologic changes of AFOP in lung tissue specimens, indicating that AFOP can be associated with lung function decline following transplantation. Although AFOP has been associated with a number of inciting factors in non-transplant patients, we could not definitively link a specific cause of declining lung allograft function with the diagnosis of AFOP for any of our patients. However, 3 of our recipients had serious infectious complications and/or episodes of allograft rejection prior to or during the onset of AFOP. We conclude that histopathologic changes consistent with AFOP can be associated with persistent post-transplant decline in allograft function; that recipients who develop AFOP may meet criteria that are consistent with a diagnosis of CLAD with clinical characteristics that can overlap with the RAS subset of CLAD, (infiltrates on HRCT, decline in FVC consistent with ventilatory restriction); that it occurs infrequently; and that its appearance is associated with a poor prognosis. Further characterisation of the AFOP phenotype of CLAD may assist efforts to prevent and/or treat this potential complication of lung transplantation.

Funding: This work was supported in part by the George and Julie Mosher Pulmonary Research Fund. The funder had no role in study design, collection and analysis of data, decision to publish, or writing of the manuscript.

Competing interests: $\mathrm{KM}$ has been an investigator in clinical trials sponsored by Abbott, Actelion, Altana, Amgen, Asthmatx, Bayer, Boehringer-Ingelheim, Bristol-Meyers Squibb, Chiron, Discovery Labs, DuPont Merck, FibroGen, Genentech, Gilead Sciences, GlaxoSmithKline, Inspire, InterMune, Johnson \& Johnson, Novartis, Nycomed, Parion
Sciences, Pfizer, Pharmaxis, PreAnalytiX, Roche, Ross, Vertex, and Wyeth. KM has also served on a clinical advisory board for InterMune and serves on a clinical trial adjudication committee for MedImmune. All authors do not report any other relevant affiliations or financial involvement with any organisation or entity with a financial interest in or financial conflict with the subject matter or materials discussed in the manuscript, apart from those disclosed.

Provenance and peer review: Not commissioned; externally peer reviewed.

Copyright: This is an open-access article distributed under the terms of the Creative Commons Attribution License, which permits unrestricted use, distribution, and reproduction in any medium, provided the original author and source are credited.

\section{References}

1. Beasley MB, Franks TJ, Galvin JR, Gochuico B, Travis WD. Acute fibrinous and organizing pneumonia: a histological pattern of lung injury and possible variant of diffuse alveolar damage. Arch Pathol Lab Med 2002;126:1064-70. PMID:12204055

2. Meyer KC, Raghu G, Verleden GM, Corris PA, Aurora $P$, Wilson KC et al.; ISHLT/ATS/ERS BOS Task Force Committee; ISHLT/ATS/ERS BOS Task Force Committee. An international ISHLT/ATS/ERS clinical practice guideline: diagnosis and management of bronchiolitis obliterans syndrome. Eur Respir J 2014;44:1479-503. http://dx.doi. org/10.1183/09031936.00107514. PMID:25359357

3. Verleden GM, Raghu G, Meyer KC, Glanville AR, Corris $P$. A new classification system for chronic lung allograft dysfunction. J Heart Lung Transplant 2014;33:12733. http://dx.doi.org/10.1016/j.healun.2013.10.022. PMID:24374027

4. Sato M, Waddell TK, Wagnetz U, Roberts HC, Hwang DM, Haroon $A$ et al. Restrictive allograft syndrome (RAS): a novel form of chronic lung allograft dysfunction. J Heart Lung Transplant 2011;30:735-42. http://dx.doi.org/10.1016/j. healun.2011.01.712. PMID:21419659

5. Sato M, Hwang DM, Waddell TK, Singer LG, Keshavjee S. Progression pattern of restrictive allograft syndrome after lung transplantation. J Heart Lung Transplant 2013;32:2330. http://dx.doi.org/10.1016/j.healun.2012.09.026. PMID:23260703

6. Paraskeva M, McLean C, Ellis S, Bailey M, Williams T, Levvey $B$ et al. Acute fibrinoid organizing pneumonia after lung transplantation. Am J Respir Crit Care Med 2013;187:13608. http://dx.doi.org/10.1164/rccm.201210-18310C. PMID:23614642

7. Yousem SA, Berry GJ, Cagle PT, Chamberlain D, Husain AN, Hruban RH et al. Revision of the 1990 working formulation for the classification of pulmonary allograft rejection: Lung Rejection Study Group. J Heart Lung Transplant 1996;15:115. PMID:8820078

8. Fukuoka J, Leslie KO. Chronic diffuse lung diseases. In Practical Pulmonary Pathology: A Diagnostic Approach. Eds. Leslie KO, Wick MR. Churchill Livingstone, Elsevier, Inc. Philadelphia, 2005. 
9. Cordier JF. Cryptogenic organising pneumonia. Eur Respir J 2006;28:422-46. http://dx.doi.org/10.1183/09031936.06. 00013505. PMID:16880372

10. Cheung O, Leslie KO. Acute lung injury. Chronic diffuse lung diseases. In Practical Pulmonary Pathology: A Diagnostic Approach. Eds. Leslie KO, Wick MR. Churchill Livingstone, Elsevier, Inc. Philadelphia, 2005.

11. Damas C, Morais A, Moura CS, Marques A. Acute fibrinous and organizing pneumonia. Rev Port Pneumol 2006;12:61520. PMID:17117329 http://dx.doi.org/10.1016/S08732159(15)30453-0

12. Kobayashi H, Sugimoto $\mathrm{C}$, Kanoh S, Motoyoshi K, Aida S. Acute fibrinous and organizing pneumonia: initial presentation as a solitary nodule. J Thorac Imaging 2005;20:291-3. http://dx.doi.org/10.1097/01.rti.0000168600.78213.85. PMID:16282908

13. Tzouvelekis A, Koutsopoulos A, Oikonomou A, Froudarakis
M, Zarogoulidis P, Steiropoulos P et al. Acute fibrinous and organising pneumonia: a case report and review of the literature. J Med Case Reports 2009;3:74. http://dx.doi. org/10.1186/1752-1947-3-74. PMID:19946550

14. Hariri LP, Unizony S, Stone J, Mino-Kenudson M, Sharma $A$, Matsubara $O$ et al. Acute fibrinous and organizing pneumonia in systemic lupus erythematosus: a case report and review of the literature. Pathol Int 2010;60:755-9. http://dx.doi.org/10.1111/j.1440-1827.2010.02586.x. PMID:20946526

15. Bhatti S, Hakeem A, Torrealba J, McMahon JP, Meyer KC. Severe acute fibrinous and organizing pneumonia (AFOP) causing ventilatory failure: successful treatment with mycophenolate mofetil and corticosteroids. Respir Med 2009;103:1764-7. http://dx.doi.org/10.1016/j. rmed.2009.07.009. PMID:19666216 